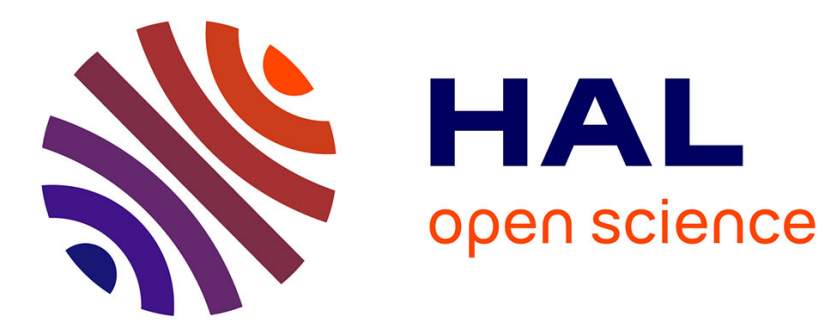

\title{
Global Measures of Local Convective Instabilities
}

Carlo Cossu, J. M Chomaz

\section{To cite this version:}

Carlo Cossu, J. M Chomaz. Global Measures of Local Convective Instabilities. Physical Review Letters, 1997, 78 (23), pp.4387-4390. hal-02042814

\section{HAL Id: hal-02042814 https://hal.science/hal-02042814}

Submitted on 20 Feb 2019

HAL is a multi-disciplinary open access archive for the deposit and dissemination of scientific research documents, whether they are published or not. The documents may come from teaching and research institutions in France or abroad, or from public or private research centers.
L'archive ouverte pluridisciplinaire HAL, est destinée au dépôt et à la diffusion de documents scientifiques de niveau recherche, publiés ou non, émanant des établissements d'enseignement et de recherche français ou étrangers, des laboratoires publics ou privés. 


\title{
Global Measures of Local Convective Instabilities
}

\author{
C. Cossu and J. M. Chomaz \\ LadHyX, CNRS UMR 156, École Polytechnique, 91128 Palaiseau Cedex, France
}

(Received 4 March 1997)

\begin{abstract}
We examine the linear stability of the Ginzburg-Landau operator with spatially varying coefficients, which mimics strongly nonparallel open flows such as wakes, jets, and boundary layers. The streamwise non-normality of the global eigenmodes explains the observed large transient growths, classically interpreted in terms of local convective instability. The use of pseudospectra provides an exact measure of spatial amplification and aids in the determination of when entrance noise dominates the open-flow dynamics. [S0031-9007(97)03331-0]
\end{abstract}

PACS numbers: 47.15.Fe, 47.20.-k, 47.27.Vf, 47.27.Wg

Wakes behind bluff bodies are known to present a Hopf bifurcation at a critical Reynolds number [1]. However, before the threshold, while the flow is globally stable, wakes are also known to exhibit large transient growths on impulsive perturbations and strong responses to small harmonic forcing. This ability to behave as spatial amplifiers has been interpreted in a Wentzel-KramersBrillouin-Jeffreys (WKBJ) framework (slowly varying wave approximation) and linked to the existence of a region where the flow is locally convectively unstable $[2,3]$. Local means that the instability is determined for the parallel flow obtained by extending to infinity the velocity profile that exists at the streamwise location $x$. The present paper, extending a work by Reddy and Trefethen [4], proposes an alternative interpretation based on the non-normality of the global modes associated with the loss of Galilean invariance and of $x \rightarrow-x$ symmetry. Concepts developed for non-normal operators such as the norm of the evolution operator and the pseudospectrum $[5,6]$ provide practical tools for quantifying the amplifying behavior of weakly or strongly nonparallel flows. These concepts apply to wakes or dynamo wave [7] below criticality as well as to mixing layers, boundary layers, or jets up to arbitrarily high Reynolds number, since these locally convectively unstable flows never become globally unstable. The same ideas are also valid when the instability breaks the $x \rightarrow-x$ symmetry, as in binary convection [8] or nonlinear optics [9].

The concept of absolute and convective instability was originally introduced in plasma physics [10] and has been successfully applied to understanding parallel openflow dynamics [3]. It applies only to parallel flows, i.e., flows invariant under translation in the streamwise direction $x$. The criterion used to discriminate between absolute and convective instability is based on the linear impulse response: i.e., the Green function $G(x, t)$, in the "laboratory" frame singled out by boundary conditions. The instability is absolute when $G(x, t)$ becomes infinite with time at any fixed location $x$ in that particular frame and convective when $G(x, t)$ goes to zero in that particular frame and to infinity in a different Galilean frame. In the laboratory frame, a convectively unstable flow will relax everywhere to the basic state as the transient is advected downstream. It will behave as a so-called spatial amplifier [3] when spatially localized harmonic forcing is applied. In contrast, in an absolutely unstable flow, a transient will initially grow in place and then saturate, leading to selfsustained oscillations.

Actual flows are not parallel since they evolve in the streamwise direction. In this case the exact stability analysis is called global as $x$ is now an eigendirection, i.e., the $x$ dependence of the eigenmode is unknown, rather than sinusoidal. When the basic flow varies on a slow spatial scale $X=\varepsilon x$ with $\varepsilon \ll 1$, the concepts developed for parallel flows apply locally at each station $X$. The objective of recent theoretical efforts is to establish a relationship between local and global instability properties. On the one hand, self-sustained resonances are well understood from both local and global points of view. In particular, the existence of a finite region of local absolute instability has been shown, using a WKBJ approximation $(\varepsilon \ll 1)$, to be necessary for global instability $[11,12]$. These mathematical results justify the physical interpretation that a self-sustained oscillation may occur through a purely hydrodynamic feedback loop consisting of two instability waves propagating downstream and upstream in the absolutely unstable region. On the other hand, the transient amplifying behavior of locally unstable but globally stable nonparallel flows has only received a local interpretation: "the flow can be thought of as a collection of spatially evolving vortical instability waves of different frequencies traveling in the downstream direction" [11]. The purpose of the present study is to develop the global point of view associated with this transient amplifier behavior. In the global approach the flow will be thought of as a collection of initially excited non-normal global modes whose amplitudes decrease in time but whose superposition produces a wave packet initially growing in time and moving in space as the relative phases of the modes vary.

To illustrate these ideas, the linearized GinzburgLandau equation is considered. This equation describes 
the wave amplitude in a bifurcating spatially extended system and has been considered to model the transition of closed [13] as well as open [14] fluid dynamical systems. It has been generalized [11] to include spatially varying coefficients that model nonparallel flows. The linear evolution of the amplitude $A(x, t)$ about the basic state $A=0$ is governed by

$$
\partial_{t} A=\mathcal{L} A \equiv-U \partial_{x} A+\mu(x) A+\gamma \partial_{x x} A,
$$

with $U$ the mean advection velocity, $\mu(x)$ the local bifurcation parameter, and $\gamma=1+i c_{d}$ the diffusion coefficient $\left(U, \mu, c_{d}\right.$ real). Boundary conditions on (1) are $A$ finite for $x= \pm \infty$.

Equation (1) governs the evolution of small perturbations $A$ to a nonparallel flow characterized by the function $\mu(x)$. If $\mu(x)$ is constant, the flow is stable for $\mu<0$, convectively unstable for $0<\mu<\mu_{t}$ with

$$
\mu_{t} \equiv U^{2} / 4|\gamma|^{2}
$$

and absolutely unstable if $\mu>\mu_{t}$.

From now on we consider quadratic variations $\mu(x)=$ $\mu_{0}+\mu_{2} x^{2} / 2$, with $\mu_{2}<0$. For a global bifurcation parameter $\mu_{0}<0$ the flow is locally stable everywhere. A finite domain of local convective instability appears for $0<\mu_{0}<\mu_{t}$, and for $\mu_{0}>\mu_{t}$ the flow becomes absolutely unstable in a portion of this domain. $\mu_{2}$ plays the role of $\epsilon$ in defining the spatial scale over which the flow is nonparallel. When $\mu_{2} \neq 0$, Eq. (1) becomes a global stability problem, solved in [15], with eigenvalues and eigenfunctions given by

$$
\begin{aligned}
\lambda_{n} & =\mu_{0}-\left(U^{2} / 4 \gamma\right)-(n+1 / 2) h, \\
\phi_{n}(x) & =\exp \left\{(U / 2 \gamma) x-\chi^{2} x^{2} / 2\right\} H_{n}(\chi x),
\end{aligned}
$$

with $h=\sqrt{-2 \mu_{2} \gamma}, \quad \chi=\left(\mu_{2} / 2 \gamma\right)^{1 / 4}, n=0,1,2, \ldots$, and $H_{n}$ the $n$th Hermite polynomial. The eigenfunction basis is orthogonal, i.e., the operator $\mathcal{L}$ is normal, only when $U=0$ or $\mu_{2}=0$. When $U$ and $\mu_{2}$ are nonzero, $\mathcal{L}$ is non-normal. The global stability is determined by the sign of the real part of the leading eigenvalue $\lambda_{0}$. The system is globally unstable if $\mu_{0}>\mu_{c}$ with

$$
\mu_{c} \equiv \mu_{t}+|h / 2| \cos [(\operatorname{Arg} \gamma) / 2] .
$$

A portion of the flow is absolutely unstable $[11,16]$ when $\mu_{0}$ exceeds $\mu_{c}$, since $\mu_{c}>\mu_{t}$. The "parallel flow" limit $\mu_{2}=0$ is singular. For $\mu_{2}$ finite, the parameter value $\mu_{c}$ at which the global bifurcation occurs is bounded away from zero since $\mu_{c}>\mu_{t}>0$, whereas when $\mu_{2}=0$ the bifurcation takes place for $\mu_{0}=0$.

This singular limit is associated with the fact that, for $0<\mu_{0}<\mu_{t}$, the smaller $\mu_{2}$, the longer and stronger are the initial transients as demonstrated by numerical simulation $[11,15]$. From the local point of view, compact perturbations will initially grow as if the flow were parallel and will start decreasing when they move away from the unstable region. To confirm this intuition by a global analysis we use Hunt's solution [16] to the nonparallel problem $\partial_{t} G-\mathcal{L} G=\delta(x-s) \delta(t)$ governing the response $G(x, s ; t)$ to an impulse applied at the location $s$ at time $t=0$. This nonparallel Green function is

$$
\begin{gathered}
G(x, s ; t)=c_{1}(t) H(t) \exp \left\{c_{2}(t)\left(x^{2}+s^{2}\right)+c_{3}(t) x s\right. \\
+U(x-s) / 2 \gamma\},
\end{gathered}
$$

with $\quad c_{1}(t)=[h / \pi \gamma \sinh (h t)]^{1 / 2} \exp \left\{\left(\mu_{0}-U^{2} / 4 \gamma\right) t\right\}$, $H$ the Heaviside function, $c_{2}(t)=-(h / 4 \gamma) \operatorname{coth}(h t)$, and $c_{3}(t)=(h / 2 \gamma)[\operatorname{coth}(h t)-\tanh (h t / 2)]$. Following [6], the transient growth may be estimated by the norm of the evolution operator $e^{\mathcal{L} t}$,

$$
\left\|e^{\mathcal{L} t}\right\| \equiv \sup _{\left\|A_{0}\right\|=1}\left\|e^{\mathcal{L} t} A_{0}\right\|
$$

which quantifies the maximum amplification over all normalized initial conditions $A_{0}(x)$ of the solution to Eq. (1) at time $t: A(x, t)=\left(e^{\mathcal{L} t} A_{0}\right)(x, t)$. This operator norm depends on the choice of function norm on the right-hand side of Eq. (6). Rather than the energy $\left(L_{2}\right)$ norm, we choose $L_{\infty}$, which measures the amplification of maximum amplitude over $x$, and which is more suitable for describing highly inhomogeneous transients. However, we have verified systematically that this choice has no qualitative impact on our results. The $L_{\infty}$ norm of the evolution operator can be computed analytically by writing $A(x, t)=$ $\left(e^{\mathcal{L} t} A_{0}\right)(x, t)=\int_{-\infty}^{\infty} G(x, s ; t) A_{0}(s) d s$, where $G$ is the Green's function given in Eq. (5), and maximizing over $A_{0}$,

$$
\begin{aligned}
\left\|e^{\mathcal{L} t}\right\|_{\infty}= & \sup _{-\infty \leq x \leq \infty} \int_{-\infty}^{\infty}|G(x, s ; t)| d s \\
= & \left|c_{1}(t)\right| \sqrt{\frac{-\pi}{c_{2 r}(t)}} \\
& \times \exp \left(\frac{U^{2}}{4|\gamma|^{2}\left[c_{3 r}(t)-2 c_{2 r}(t)\right]}\right),
\end{aligned}
$$

where $c_{2 r}(t)$ and $c_{3 r}(t)$ denote the real parts of the functions $c_{2}(t)$ and $c_{3}(t)$.

Figure 1 shows the time evolution of $\left\|e^{\mathcal{L} t}\right\|$ for various values of $\mu_{0}$. By definition (6), $\left\|e^{\mathcal{L} t}\right\|=1$ for $t=0$. Large-time expansion of (7) gives $\left\|e^{\mathcal{L} t}\right\| \sim \exp \left\{\left(\mu_{0}-\right.\right.$ $\left.\left.\mu_{c}\right) t\right\}$ which grows exponentially when $\mu_{0}>\mu_{c}$, i.e., when the system is globally unstable (curve $e$ ). When $\mu_{0}=\mu_{c},\left\|e^{\mathcal{L} t}\right\|$ saturates (curve $d$ ). When $\mu_{0}<\mu_{c}$, $\left\|e^{\mathcal{L} t}\right\|$ decays at large times (curves $a, b, c$ ) but presents a transient growth only when $\mu_{0}>0$, i.e., when the system is locally unstable (curve $c$ ). For the present moderate value of $\mu_{2}$, i.e., strong nonparallelism, it is surprising that the local instability still has physical implications and induces extremely large transient growth exceeding $10^{5}$ for $\mu_{0}$ close to $\mu_{c}$. We define the total growth $M$ as the maximum of the curve $\left\|e^{\mathcal{L} t}\right\|$ (Fig. 1),

$$
M\left(\mu_{0}, \mu_{2}, U, \gamma\right)=\sup _{t \geq 0}\left\|e^{\mathcal{L} t}\right\| .
$$




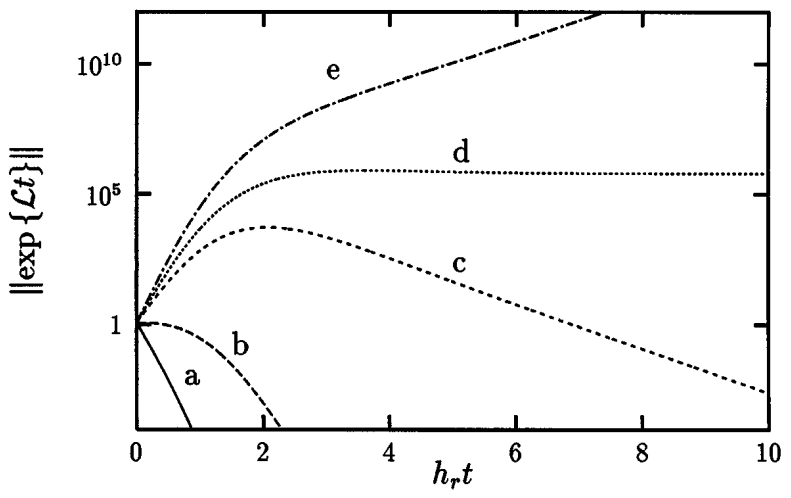

FIG. 1. Norm of the evolution operator $\left\|e^{\mathcal{L} t}\right\|_{\infty}$ versus time for $\mu_{2}=-0.1, \gamma=1-i, U=6$, and curve $a \mu_{0}<0$, curve $b \mu_{0}=0$, curve $c 0<\mu_{0}<\mu_{c}$, curve $d \mu_{0}=\mu_{c}$, and curve $e \mu_{0}>\mu_{c}$.

Figure 2 presents the contours of $M$ in the $\left(\mu_{0}, \mu_{2}\right)$ space for the same parameters as Fig. 1. In the globally unstable (dark gray) region of the parameter space $\left[\mu_{0} \geq \mu_{c}\left(\mu_{2}, U, \gamma\right)\right], M$ is infinite. In the globally stable region, $M$ is finite. For $\mu_{0}>0, M$ diverges as $\mu_{2}$ goes to zero, indicating that the parallel flow limit is singular. For small but finite $\mu_{2}$, we have $M \gg 1$ if a locally unstable region is present in the flow $\left(\mu_{0}>0\right)$, whereas we have $M=1$ when the flow is everywhere locally stable $\left(\mu_{0}<0\right)$. From Fig. 2, for strongly nonparallel flows (large $\mu_{2}$ ) the evolution of $M$ follows only broadly the local instability criteria. However, it is remarkable that, for $\mu_{2}$ as large as -0.1 , a $10^{5}$ transient growth in amplitude may be experienced by the perturbation although the flow is globally stable. Therefore we claim that the quantity $M$ (which is global and does not depend on the WKBJ approximation) quantifies the transient amplifying behavior of a locally convectively un-

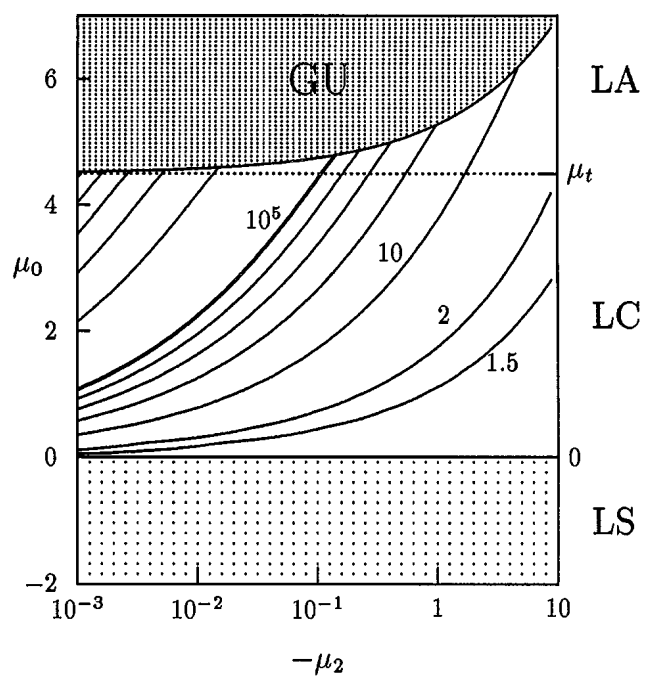

FIG. 2. Total growth $M$ as a function of $\mu_{2}$ and $\mu_{0}$ with $\gamma=1-i, U=6 . \quad M=1$ in the light gray (LS) region; $M=\infty$ in the globally unstable (GU) region (dark gray) limited by the $\mu_{c}\left(\mu_{2}\right)$ curve. The contour levels are $1.5,2,10,10^{2}$, $\ldots, 10^{5}$ (boldfaced solid), $10^{15}, 10^{25}, 10^{35}, 10^{45}$. stable flow, independent of its degree of nonparallelism. Similarly, we now wish to characterize the noise amplifying behavior of globally stable open flows using the recently popularized tools of non-normal operators ([6] and references therein). The response of the flow to a forcing term $-\tilde{f}(x) e^{\sigma t}$ is $A=\tilde{A}(x) e^{\sigma t}$ where $[\mathcal{L}-\sigma I] \tilde{A}=\tilde{f}$ and $\sigma$ is complex ( $I$ is the identity operator). This problem is formally solved by introducing the resolvent operator $R(\sigma) \equiv[\mathcal{L}-\sigma I]^{-1}$, so that $\tilde{A}=R(\sigma) \tilde{f}$. The resolvent norm $\|R(\sigma)\| \equiv \sup _{\|\tilde{f}\|=1}\|R(\sigma) \tilde{f}\|$ is infinite on the spectrum of $\mathcal{L}$. When $\mathcal{L}$ is normal, $\|R(\sigma)\|$ decays strongly with the distance between $\sigma$ and the spectrum of $\mathcal{L}$ (the decay is inversely proportional to this distance if the energy norm is used). The decay may no longer be strong when the operator is non-normal and $\|R(\sigma)\|$ may then retain extremely large values even far from the spectrum. $\|R(\sigma)\|$ is related to the pseudospectrum which has been the focus of much recent interest [6]: the $\epsilon$ pseudospectrum of $\mathcal{L}$ is the set of $\sigma$ such that $\|R(\sigma)\| \geq \epsilon^{-1}$.

Figure 3 presents numerical computations [17] of the boundaries of the pseudospectra of the operator $\mathcal{L}$ for $\mu_{0}=0$, i.e., contours of the resolvent norm $\|R(\sigma)\|$. Two cases are considered: a highly nonparallel flow [Fig. 3(a)] with $\mu_{2}=-0.1$ and a weakly nonparallel flow [Fig. 3(b)] with $\mu_{2}=-0.001$. The spectrum $\left(\lambda_{n}\right)$ is also plotted on Fig. 3 as a set of crosses. In the highly nonparallel case of Fig. 3(a), the rightmost point of the $\|R(\sigma)\|=10^{5}$ contour is close to the rightmost cross, i.e., the leading eigenvalue $\lambda_{0}$. In contrast, in the weakly nonparallel case of Fig. 3(b), the $\|R(\sigma)\|=10^{5}$ contour is far from the spectrum. This shows that $\mathcal{L}$ becomes increasingly non-normal as the flow becomes more parallel ( $\mu_{2}$ small). Once again, the limit $\mu_{2}=0$ is singular. When $\mu_{2} \rightarrow 0$, the resolvent norm $\|R(\sigma)\|$ tends to infinity exponentially with $1 /\left|\mu_{2}\right|$ for all $\sigma$ inside a critical curve [the dashed curves in Figs. 3(a) and 3(b)]. This critical curve is the (continuous) spectrum of $\mathcal{L}$ for $\mu_{2}=0$, i.e., the temporal branch for the instability of the parallel flow. In other words, as $\mu_{2} \rightarrow 0$, an $\epsilon$-pseudospectrum boundary for $\epsilon$ small approaches the spectrum of $\mathcal{L}$ for $\mu_{2}=0$, whereas the spectrum itself does not. Similar behavior was observed in [4] for a convection-diffusion operator on an interval. The pseudospectrum (but not the spectrum) for the inhomogeneous system-our nonparallel flow, and the finite interval of [4] - approaches the spectrum of the homogeneous system-our parallel flow and the infinite line of [4]. However, this behavior was not interpreted in terms of local instability.

The above discussion pertains to a single value of the global bifurcation parameter: $\mu_{0}=0$. We now examine the effect of varying $\mu_{0}$. Although, in general, the resolvent norm $\|R(\sigma)\|$ depends in a complicated manner on the parameters of a problem, in our case, $\|R(\sigma)\|$ can be obtained for any value of $\mu_{0}$ merely by translating $\sigma_{r}$ via $\left\|R\left(\sigma, \mu_{0}\right)\right\|=\left\|R\left(\sigma-\mu_{0}, 0\right)\right\|$. Hence Fig. 3 can be 
a)

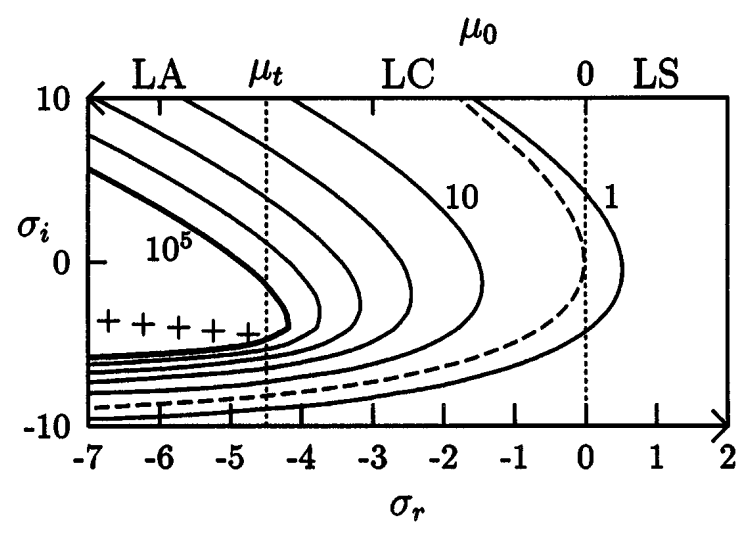

b)

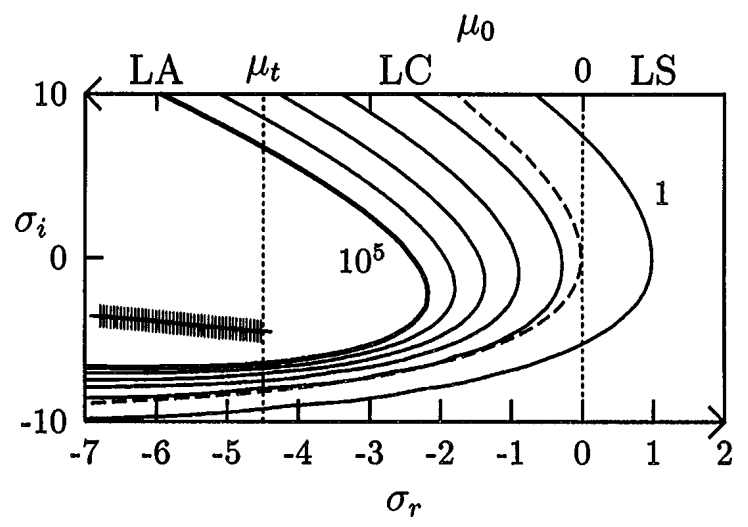

FIG. 3. Spectrum $(+)$ and $L_{\infty} \epsilon$-pseudospectrum boundaries (solid lines) in the complex $\sigma$ plane. (a) $\mu_{2}=-0.1$, and (b) $\mu_{2}=-0.001$ both with $\mu_{0}=0, \gamma=1-i, U=6$. The $\epsilon^{-1}$ values are from right to left at the levels $10^{0}, \ldots, 10^{4}$ (solid) and $10^{5}$ (boldfaced solid). The dashed curve is the spectrum (temporal branch) in the parallel case $\left(\mu_{2}=0\right)$. The $\mu_{0}$ upper axis allows both figures to be viewed as bifurcation diagrams.

reinterpreted, with the help of the upper $\mu_{0}$ axis, as displaying $\left\|R\left(\sigma=i \Omega, \mu_{0}\right)\right\|=\left\|R\left(-\mu_{0}+i \Omega, 0\right)\right\|$ for the single value $\sigma_{r}=0$. Considering pure imaginary values $\sigma=i \Omega$ corresponds to harmonic forcing, which has the physical interest of representing experimental actuators (loudspeakers, vibrating ribbons, ...) and experimental noise imposed by imperfections of the apparatus. When $0<\mu_{0}<\mu_{t}$, i.e., when a spatial domain is locally convectively unstable, the smaller $\mu_{2}$, the larger the maximum amplification over all $\Omega$. This amplification becomes infinite at the global bifurcation threshold $\mu_{0}=\mu_{c}$, i.e., when the rightmost eigenvalue crosses the imaginary axis and a finite spatial domain is locally absolutely unstable. If the imposed noise is of amplitude $10^{-5}$, we will obtain an order 1 response when $\mu_{0}$ crosses the contour $\|R(\sigma)\|=10^{5}$ [boldfaced curve in Figs. 3(a) and 3(b)]. A similar amplification is thought to induce a noise-sustained bifurcation [6,18] in plane Poiseuille and Couette flows.

Previous analyses have considered non-normality only in the cross-stream direction. In this Letter, we have studied non-normality in the streamwise direction, and related it to local instability. The present analysis can quantify the receptivity (e.g., the ability to amplify external perturbations) of locally unstable flows and may explain why some globally stable flows are observed apparently unperturbed (e.g., the wake of a circular cylinder for $\operatorname{Re}<$ 47) and others always present irregular structures (mixing layers or jets). A complete analysis of these hydrodynamic instabilities should combine the effect of non-normality in both cross-stream and streamwise directions. An ideal test case for these combined effects could be a nonparallel (i.e., streamwise) perturbation of Poiseuille or Couette flows.

We greatly thank L. Tuckerman for her assistance and helpful comments and G. Giolitti for stimulating research by providing inspiring "gelati." We acknowledge the computational facilities provided by the Institut du Développement et des Ressources en Informatique (IDRIS/CNRS) under Grant No. 960761/CP6.

[1] C. Mathis, M. Provansal, and L. Boyer, J. Phys. (Paris), Lett. 45, 483 (1984).

[2] M. Gaster, Phys. Fluids 11, 723 (1968).

[3] P. Huerre and P. A. Monkewitz, Annu. Rev. Fluid Mech. 22, 473 (1990).

[4] S. C. Reddy and L. N. Trefethen, SIAM J. Appl. Math. 54, 1634 (1994).

[5] H. J. Landau, J. d'Analyse Math. 28, 335 (1975).

[6] L. N. Trefethen, A.E. Trefethen, S.C. Reddy, and T. A. Driscoll, Science 261, 578 (1993).

[7] A. M. Soward, Geophys. Astrophys. Fluid Dyn. 64, 201 (1992).

[8] P. Kolodner, Phys. Rev. Lett. 66, 1165 (1991); M. C. Cross, Phys. Rev. Lett. 57, 93 (1986).

[9] P. Coullet, L. Gil, and R. Repoux, Phys. Rev. Lett. 62, 2957 (1989); P. Coullet, L. Gil, and F. Rocca, Opt. Commun. 73, 40 (1989).

[10] R. J. Briggs, Electron-Stream Interaction with Plasmas (MIT Press, Cambridge, MA, 1964); A. Bers, in Physique des Plasmas, edited by C. D. Witt and J. Peyraud (Gordon \& Breach, New York, 1975), pp. 117-225.

[11] J. M. Chomaz, P. Huerre, and L. G. Redekopp, Phys. Rev. Lett. 60, 25 (1988).

[12] P. Monkewitz, P. Huerre, and J. Chomaz, J. Fluid Mech. 251, 1 (1993).

[13] A. C. Newell and J. A. Whitehead, J. Fluid Mech. 38, 279 (1969).

[14] K. Stewartson and J. T. Stuart, J. Fluid Mech. 48, 529 (1971).

[15] J. M. Chomaz, P. Huerre, and L. G. Redekopp, in Proceedings of the Sixth Symposium on Turbulent Shear Flows, Toulouse, France, 1987 (Paul Sabatier University, Toulouse, 1987), pp. 3.2.1-6.

[16] R.E. Hunt, Ph.D. thesis, Trinity College, Cambridge, 1995; R. E. Hunt and D. G. Crighton, Proc. R. Soc. London A 435, 109 (1991).

[17] The discretized operator was obtained via centered finite differences with homogeneous boundary conditions (Dirichlet at the left and Neumann at the right boundary).

[18] L. H. Gustavsson, J. Fluid Mech. 224, 241 (1991); K. M. Butler and B. F. Farrell, Phys. Fluids A 4, 1637 (1992). 